\title{
CURCUMIN MONO-CARBONYL ANALOGS AS POTENT ANTIBACTERIAL COMPOUNDS: SYNTHESIS, BIOLOGICAL EVALUATION AND DOCKING SIMULATION STUDY
}

\author{
B. Wijianto ${ }^{1,3}$, Ritmaleni ${ }^{1, *}$, H. Purnomo ${ }^{1}$ and A. Nurrochmad ${ }^{2}$ \\ ${ }^{1}$ Department of Pharmaceutical Chemistry, Universitas Gadjah Mada, \\ Yogyakarta-55281, Indonesia \\ ${ }^{2}$ Department of Pharmacology, Universitas Gadjah Mada, Yogyakarta-55281, Indonesia \\ ${ }^{3}$ Department Pharmaceutical Chemistry, Universitas Tanjungpura, Pontianak-78115, Indonesia \\ *E-mail: ritmaleni@ugm.ac.id
}

\begin{abstract}
The present study aims to synthesize, evaluation of biological activity, and study docking of new analog compounds A01, B01, C01, and C02 as antibacterial. Claisen-Schmidt method used to synthesize A01, B01, C01, and $\mathrm{C} 02$ compounds. The new analog compounds $\mathrm{A} 01, \mathrm{~B} 01, \mathrm{C} 01$, and $\mathrm{C} 02$ have antibacterial activity with $\mathrm{IC}_{50}$ values of $37.1 \mu \mathrm{M}, 140 \mu \mathrm{M}$, respectively; $79 \mu \mathrm{M}$ and $117 \mu \mathrm{M}$ in the aureus; $56.6 \mu \mathrm{M}, 282 \mu \mathrm{M}, 97.6 \mu \mathrm{M}$ and $186.1 \mu \mathrm{M}$ in subtilis; $291.2 \mu \mathrm{M}, 1025.9 \mu \mathrm{M}, 679.81 \mu \mathrm{M}$ and $561.9 \mu \mathrm{M}$ in E.coli; $29.5 \mu \mathrm{M}, 310.5 \mu \mathrm{M}, 32.9 \mu \mathrm{M}$ and $130.6 \mu \mathrm{M}$ in P. aeruginosa; $66.6 \mu \mathrm{M}, 328.6 \mu \mathrm{M}, 49.3 \mu \mathrm{M}$ and $253.8 \mu \mathrm{M}$ in mutans; $97.2 \mu \mathrm{M}, 392.2 \mu \mathrm{M}, 129.6 \mu \mathrm{M}$, and $191.6 \mu \mathrm{M}$ in faecialis; $44,811 \mu \mathrm{M}$ (A113) in C.albicans. Synthesized mono-carbonyl curcumin analog compounds have antibacterial activity through inhibition of bacterial cell wall synthesis.
\end{abstract}

Keywords: Mono-carbonyl Analogs of Curcumin, in silico, Antibacterial

(c) RASĀYAN. All rights reserved

\section{INTRODUCTION}

Surveillance results from the World Health Organization (WHO) in 2016 found that nearly 50,000 people, including men, women, and children around the world, die every day due to microbial infections. ${ }^{1}$ A report released by WHO states that 16 million people died in 1990 due to microbial infection. In 2010 the number was reduced to 15 million and was estimated to be 13 million in 2050. These data show that the death rate due to microbial infections decreased during the 1990 period but was very slow, and predicted to be 13 million in 2050. Decrease happens not because the effectiveness of antibiotics is, however, caused by a sudden population explosion and antimicrobial resistance. ${ }^{2-4}$ Incidence of resistance becomes a severe problem globally that requires the attention and action of all communities and stakeholders in effective prevention and treatment. The success rate of major surgery and cancer chemotherapy will be low if without effective antibiotics. Besides, patients in antibiotic-resistant cases will incur higher health care costs compared to patients without antibiotic-resistant cases because of the longer duration of the disease, additional laboratory tests, and the use of more expensive drugs. The use of antibiotics in farming also contributes significantly to AMR cases in addition to the irrational use of drugs by patients.

The mono-carbonyl of curcumin compound is an analog compound of curcumin. The presence of six rings formed by binding of hydrogen in the basic framework is a potential substrate for Aldo-keto reductase enzymes in the human body, thus increasing the metabolic processes in the body. The structure chemical analogs mono-carbonyl of curcumin has recently performed from curcumin exhibiting vigorous antimicrobial activity with increased solubility and stability A-C series ${ }^{5-8}$ (Fig.-1).

Analogs Mono-carbonyl of curcumin mainly containing hydroxyl groups in the para (R4) and two chlorine groups in the ortho position (R2 and R6) on the aromatic ring, with six-ring (series-A) ketones, five-ring (Series-B) and aliphatic-ketones (C-series) known to have anti-inflammatory, antioxidant and antibacterial activities. ${ }^{9}$

Rasayan J. Chem., 13(1), 1153-1165(2020)

http://dx.doi.org/10.31788/RJC.2020.1325554

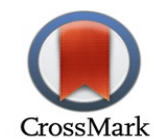


RASĀYAN J. Chem.

Vol. 13 | No. 2 |1153 - 1165| April - June | 2020

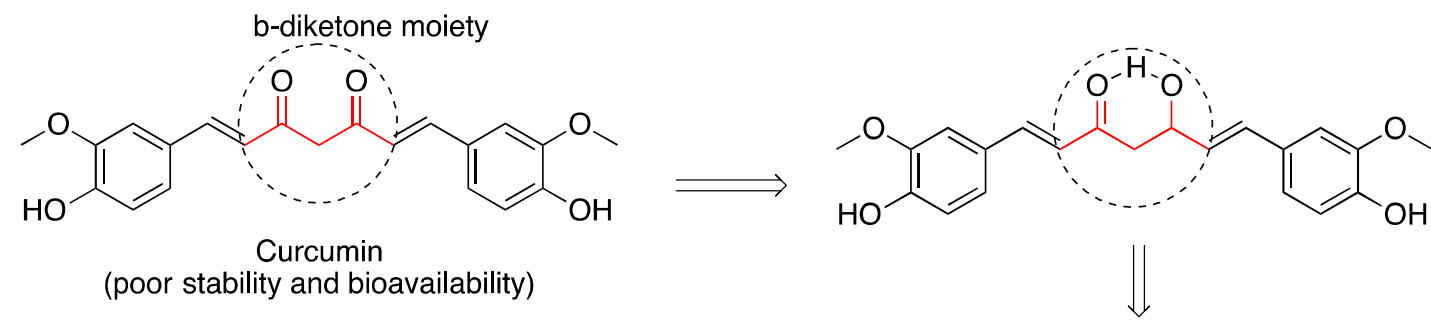

Substrate for aldo-keto reductase

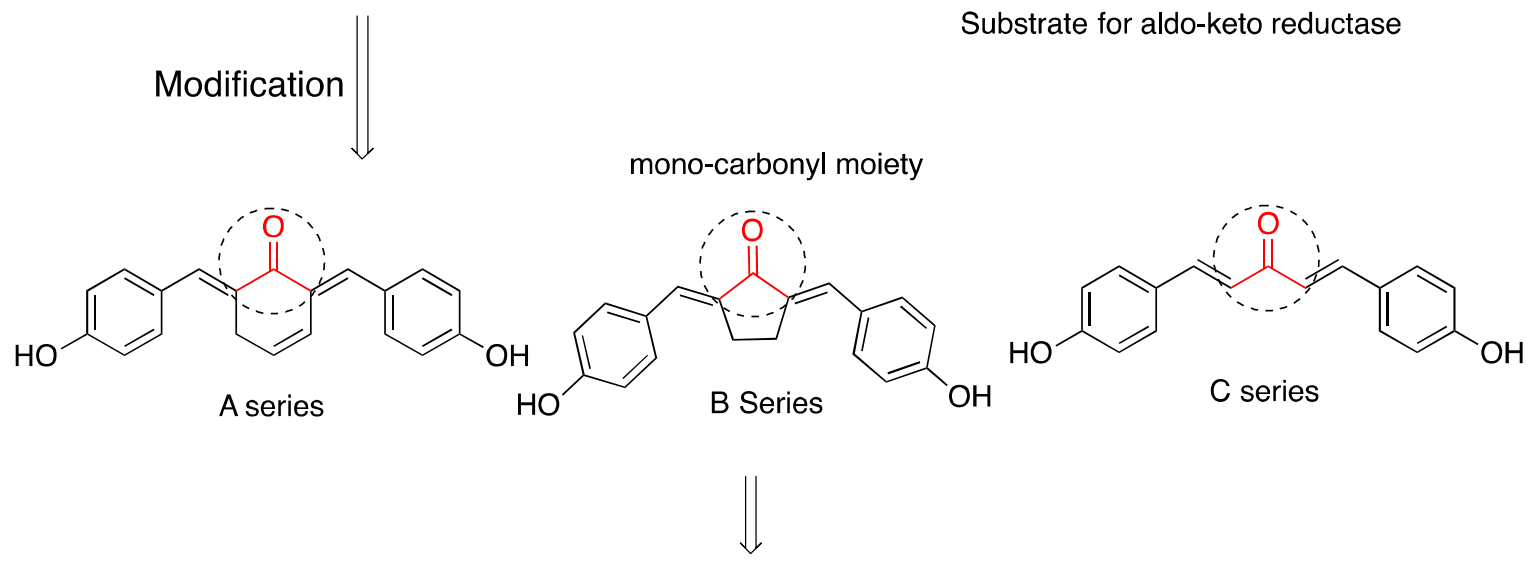

high potency and good bioavailability

Fig.-1: Chemical Structure of Mono-carbonyl Analogs of Curcumin

Analogs mono-carbonyl of curcumin can be formed from synthesis by the Claisen-Schmidt condensation method. ${ }^{6,9-14}$ The Claisen-Schmidt reaction occurs between aromatic aldehyde derivatives and acetophenone derivatives under acidic and basic conditions. ${ }^{15}$ The Claisen-Schmidt method is the most popular and the chosen one because easy to react and pure, efficient raw material, and safe for the environment because it can be done with little or without solvent. Claisen and Schmidt first reported this reaction in $1881 .{ }^{16}$ The Claisen-Schmidt reaction can be carried out through a grinding process without the use of solvents and irradiation by microwave. Apart from the above reasons, another attraction of this method is the vast selection of ketones and aldehydes, which have various functional groups as starting material. For consideration of global health problems arising from antimicrobial resistance and the urgent need for potential antimicrobial agents, this study targets the synthesis of new analogs of mono-carbonyl curcumin and their evaluation of various bacterial strains. The inhibition zone and MIC of all the synthesized compounds were determined. Furthermore, various binding interactions of potential compounds are studied using molecular docking.

\section{Chemicals}

\section{EXPERIMENTAL}

3,5-dibromo-4-hydroxybenzaldehyde, 3-hydroxybenzaldehyde, 2-Chloro-6-fluorobenzaldehyde, 3Bromo-4-methoxybenzaldehyde, cyclohexanone, cyclopentanone, acetone, tetrahydrofuran, Hydrochloric acid, ethanol, ethyl acetate, methanol, Dimethyl sulfoxide (DMSO), amoxicillin, chloramphenicol, kanamycin, and streptomycin acquired from Sigma-Merck. The bacterial tests used in this study were strains of the bacteria Staphylococcus aureus ATCC 25923, Bacillus subtilis ATCC 6633, Escherichia coli ATCC 25922, Enterococcus faecialis ATCC 29212, Streptococcus mutans ATCC 25175, Pseudomonas aeruginosa ATCC 27853, and Candida albicans ATCC 10231. Bacterial growth media used Brain Heart Infusion (BHI) and Mueller Hinton Agar (MHA), while the yeast growing media used in the test were sabouraud dextrose agar (SDA) and sabouraud dextrose broth (SDB) obtained from Merck and oxoid. 
RASĀYAN J. Chem.

Vol. 13 | No. 2 |1153 - 1165| April - June | 2020

\section{Instrument}

PC with Intel Core i3-6006U $2.0 \mathrm{GHz}, 4 \mathrm{~GB}$ and Windows 7 as the operating system. BUCHI Melting Point B-540 with a temperature gradient at $5^{\circ} \mathrm{C} / \mathrm{min}$ was used for the melting point test. The purity of the synthesized compound was measured using HPLC Elite La-Chrome ${ }^{\circledR}$. JEOL ${ }^{\circledR}$ spectrophotometer 500 $\mathrm{MHz}$ was used to measure the 1H-NMR Spectrum. the antibacterial assay used autoclaves, Laminar airflow (LAF), analytical scales Shimadzu AUW220 ${ }^{\circledR}$, glassware, 96 well Iwaki ${ }^{\circledR}$ microplate, Socorex ${ }^{\circledR}$ micropipette, cuvet, vortex, ose, bunsen, blue and yellow tip, spectrophotometer Optima SP-3000nano, Elisa reader, incubator and refrigerator.

\section{Synthesis of A01, B01, C01 and C02}

In each round bottom flask added $3.572 \mathrm{mmol}$ of 3,5-dibromo-4-hydroxybenzaldehyde and $1.786 \mathrm{mmol}$ of cyclohexanone; $8.188 \mathrm{mmol}$ of 3-hydroxybenzaldehyde and $3.009 \mathrm{mmol}$ of cyclopentanone; 4.975 mmol of 3-Bromo-4-hydroxybenzaldehyde and $2.487 \mathrm{mmol}$ of acetone. $6.306 \mathrm{mmol}$ of 2-Chloro-6fluorobenzaldehyde and $3.153 \mathrm{mmol}$ of acetone. $2 \mathrm{ml}$ of THF and $0.2 \mathrm{ml}$ of concentrated hydrochloric acid were added to each flask and stirred at room temperature for 2 hours. Then, the temperature raised to $40-50 \mathrm{oC}$, and stirring was continued for 8 hours until the reaction was complete. The crude product was washed out by ethanol: cold water (1: 1) then filtered by Buchner. The second washed for residue was performed by different ratios of ethanol and cold water (3: 2) until $\mathrm{pH} 7-8$ reached out. The residue was filtered and dried in the oven. Recrystallization performed by dissolving the residue in acetone, then cold water was added until a precipitate formed. TLC and melting point tests were carried out to find out the purity of the product qualitatively.

\section{Purity Test by HPLC}

Analysis purity test of the product synthesis using HPLC, as a comparison used starting material (aldehydes). Synthesis products and starting material analyzed at $100 \mathrm{ppm}$ concentration. Observations determined at UV wavelength $350 \mathrm{~nm}$ and a mobile phase of acetonitrile: water (80:20) with a flow rate of $1 \mathrm{ml} / \mathrm{min}, 100 \mathrm{psi}$ pressure, and $20 \mu \mathrm{L}$ volume injection in column $\mathrm{C}_{18}$.

Table-1: HPLC Purity Test Results

\begin{tabular}{c|c|c}
\hline Compound & Retention Time (min) & Area (\%) \\
\hline A01 & 3.64 & 99.999 \\
Starting material of A01 & 1.41 & 99.779 \\
\hline B01 & 1.30 & 99.999 \\
Starting material of B01 & 1.84 & 100.00 \\
\hline C01 & 1.32 & 99.999 \\
Starting material of C01 & 1.29 & 85.071 \\
\hline C02 & 2.21 & 99.999 \\
Starting material of C02 & 1.61 & 74.690
\end{tabular}

The different retention times of each compound (Table-1) indicate the real yield of the synthesized compound. So it known that each compound could be adequately separated using the HPLC system. The results showed that the synthesized compound was pure, which was confirmed using the respective starting material.

\section{Molecular Modeling}

All mono-carbonyl structures of curcumin depicted in 2D in the MOE 'builder' menu, then optimized using MOPAC. The AM-1 semi-empirical method with a $0.01 \mathrm{RMS} \mathrm{kcal} / \mathrm{mol} / \mathrm{A}^{\wedge} 2$ gradients is used to determine quantum chemical algorithms.

\section{Docking Studies}

In silico study was conducted to known antibacterial activity through $1 \mathrm{PCW}$ as protein test, which is a protein from D-Alanyl-D-Alanine Carboxypeptidase and 3N7W, which is a protein from beta-lactamase that available in the protein data bank. Protein validation and docking process were performed by using 
RASĀYAN J. Chem.

Vol. 13 | No. 2 |1153 - 1165| April - June | 2020

alpha PMI method on placement and induced fit on refinement scoring by GBVI/WSA dG as protocols. Protein validated was selected based on RMSD (Root Mean Square Deviation) value. ${ }^{17}$<smiles>O=C1CCCCC1</smiles><smiles>O=Cc1cccc(O)c1</smiles><smiles>[R8]c1c([R6])c([2H])c([R8])c(C=O)c1[R8]</smiles>

$01=\mathbf{R}_{4}, \mathbf{R}_{3}=\mathrm{Br}, \mathrm{OH}$

$02=R_{1}, R_{5}=\mathrm{Cl}, \mathrm{F}$<smiles>O=C1/C(=C/c2cc(Br)c(O)c(Br)c2)CCC/C1=C\c1cc(Br)c(O)c(Br)c1</smiles>

A01<smiles>O=C1/C(=C/c2cccc(O)c2)CC/C1=C\c1cccc(O)c1</smiles>

B01

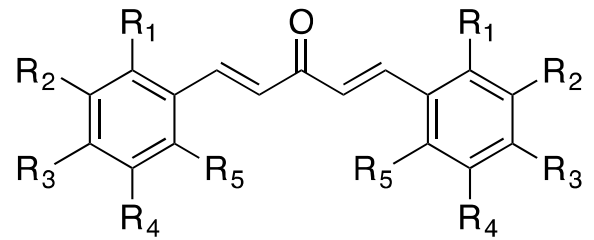

C01-02

Scheme-1: Reagent and Condition of Synthesis: a- THF and $\mathrm{HCl} ; 8 \mathrm{~h}$ and $50^{\circ} \mathrm{C}$

\section{2,6-bis-(3',5'-dibromo, 4'- hydroxybenzylidene)-cyclohexanone (A01)}

Yellow powder, yield $53 \%, \mathrm{mp} 239.8-240.6{ }^{\circ} \mathrm{C}$; $\mathrm{Rf}=0.825$, ethyl acetate: $\mathrm{CHCl}_{3}(1: 20)$; IR $\gamma(\mathrm{cm}-1)(\mathrm{KBr})$ : 3199.11 (-OH bonded), 2935.18 (=C-H stretching aromatic), 2863.79 (-C-H stretching aliphatic), 1657.48 $\left(\mathrm{C}=\mathrm{C}\right.$ stretching aliphatic), 1599.47 ( $\mathrm{C}=\mathrm{O}$ stretching $\alpha \beta, \alpha^{\prime} \beta^{\prime}$-un-sat), $1474.27(\mathrm{C}=\mathrm{C}$ stretching aromatic), 1399.82 (C-H bending aliphatic), 1265.49 (C-CO-C coupled stretching and bending), 1142.89 (C-OH stretching), 719.93 (C-Br stretching), MS (EI-MS, m/z ): $592\left[\mathrm{M}^{+}+2 \mathrm{H}\right] ; 42$ (base peak) $\left[\mathrm{M}^{+\cdot}\right] ; 592,543$, 515, 486, 342, 302, 274, 209, 190, 144, 115, 101, dan 42; ${ }^{1} \mathrm{HNMR}\left(500 \mathrm{MHz}, \mathrm{ppm}, \mathrm{CDCl}_{3}\right.$-d6) $\delta 1.858$ $\left(2 \mathrm{H}, q u i, \mathrm{H} 10, \mathrm{CH} 2\right.$ cyclohexanone), $\delta 2.965\left(4 \mathrm{H}, q u i, \mathrm{H} 9,11, \mathrm{CH}_{2}\right.$ cyclohexanone), $\delta 7.531\left(2 \mathrm{H}, s, \mathrm{H}_{7}, 14\right.$, $\mathrm{CH}$-aliphatic), $\delta 7.729$ (4H, $\left.s, \mathrm{H}_{4,6,16,20} \mathrm{Ar}-\mathrm{CH}\right), \delta 8.978$ (2H, $\left.s, \mathrm{Ar}-\mathrm{OH}\right) .{ }^{13} \mathrm{C}-\mathrm{NMR}(125 \mathrm{MHz}, \mathrm{ppm}$, $\left.\mathrm{CDCl}_{3}\right): \delta 22.844, \delta 28.421, \delta 110.125, \delta 134.064, \delta 134.265, \delta 136.348, \delta 149.835, \delta 189.333$.

\section{2,5-bis-(3'-hydroxybenzylidene)-cyclopentanone (B01)}

Yellow powder, yield $53.7 \%, \mathrm{mp} 119.4-120.1^{\circ} \mathrm{C}$; Rf $=0.61$, ethyl acetate : $\mathrm{n}-\mathrm{hex}(1: 7)$; IR $\gamma(\mathrm{cm}-1)(\mathrm{KBr})$ : 3401,49 (-OH bonded), 3068.39 (=C-H stretching aromatic), 2919.36 (-C-H stretching aliphatic), 1651.06 $\left(\mathrm{C}=\mathrm{C}\right.$ stretching aliphatic), 1619,61 $\left(\mathrm{C}=\mathrm{O}\right.$ stretching $\alpha \beta, \alpha^{\prime} \beta^{\prime}$-un-sat $), 1450.22(\mathrm{C}=\mathrm{C}$ stretching aromatic $)$, 1345.96 (C-H bending aliphatic), 1284.78 (C-CO-C coupled stretching and bending), 674.82; 782.86; 880.89 (Benzene substituted meta); MS (EI-MS, m/z ): $291\left[\mathrm{M}^{+}+2 \mathrm{H}\right] ; 291$ (base peak) $\left[\mathrm{M}^{+{ }^{-}}\right] ; 291,275$, 263, 247, 225, 185, 157, 131, 128, 103, 89, 77, 51; ${ }^{1} \mathrm{HNMR}\left(500 \mathrm{MHz}, \mathrm{ppm}, \mathrm{CDCl}_{3}\right.$-d6) $\delta 2.055(4 \mathrm{H}, q$, C9,10, CH2 cyclopentanone), $\delta 6.884(2 \mathrm{H}, d d, J 1=6.5, J 2=1.5 \mathrm{~Hz}, \mathrm{C} 4,15, \mathrm{Ar}-\mathrm{CH}), \delta 7.122(2 \mathrm{H}, d$, $J=8 \mathrm{~Hz}, \mathrm{C} 2,17, \mathrm{Ar}-\mathrm{CH}), \delta 7.277(2 \mathrm{H}, d, \mathrm{C} 4,15, \mathrm{Ar}-\mathrm{CH}), \delta 7.292(2 \mathrm{H}, d d, J 1=J 2=8 \mathrm{~Hz}, \mathrm{C} 3,16, \mathrm{Ar}-$ $\mathrm{CH}), \delta 7.379(2 \mathrm{H}, s, \mathrm{C} 6,19, \mathrm{CH}$ aliphatic), $\delta 7.994(2 \mathrm{H}, s, \mathrm{C} 7,13, \mathrm{CH}$ aliphatic) dan $\delta 9.667(2 \mathrm{H}, s, \mathrm{Ar}-$ 
RASĀYAN J. Chem.

Vol. 13 | No. 2 |1153 - 1165| April - June | 2020

$\mathrm{OH}) .{ }^{13} \mathrm{C}-\mathrm{NMR}\left(125 \mathrm{MHz}, \mathrm{ppm}, \mathrm{CDCl}_{3}\right): \delta 26.973, \delta 117.719, \delta 117.950, \delta 122.970, \delta 130.687, \delta 134.114$, $\delta 137.685, \delta 138.433, \delta 158.581, \delta 196.870$.

\section{1,5-bis-(3'-Bromo-4'-hydroxybenzylidene)-1,4-pentadien-3-one (C01)}

Reddish powder, yield $32.6 \%, \mathrm{mp} 212.2-213.4^{\circ} \mathrm{C}$; $\mathrm{Rf}=0.425$, ethyl acetate : $\mathrm{n}$-hex(1:7); IR $\gamma(\mathrm{cm}-1)$ (KBr): 3377.71 (-OH bonded), 2949.35 (=C-H stretching aromatic), 2861.23 (-C-H stretching aliphatic), $1673.86\left(\mathrm{C}=\mathrm{C}\right.$ stretching aliphatic), $1643.69\left(\mathrm{C}=\mathrm{O}\right.$ stretching $\alpha \beta, \alpha^{\prime} \beta$ '-un-sat $), 1494.10(\mathrm{C}=\mathrm{C}$ stretching aromatic), 1355,29 (C-H bending aliphatic), 1269.40 (C-CO-C coupled stretching and bending), 1199.96 (C-OH stretching), 720.14 (C-Br stretching); MS (EI-MS, m/z ): $424\left[\mathrm{M}^{+}+2 \mathrm{H}\right] ; 118$ (base peak) $\left[\mathrm{M}^{+\cdot}\right]$; 442, 343, 316, 263, 236, 225, 198, 171, 146, 118, 39; ${ }^{1} \mathrm{HNMR}\left(500 \mathrm{MHz}, \mathrm{ppm}, \mathrm{CDCl}_{3} \mathrm{-d} 6\right): 6.948$ (2H, $d$, $J=8.5 \mathrm{~Hz}, \mathrm{C} 1,16, \mathrm{Ar}-\mathrm{CH}), 7.103(2 \mathrm{H}, d, J=15.5 \mathrm{~Hz}, \mathrm{C} 8,10$, Aliphatic- $\mathrm{CH}), 7.556(2 \mathrm{H}, d d, J 1=J 2=2 \mathrm{~Hz}$, $\mathrm{C} 4,13, \mathrm{Ar}-\mathrm{CH}) ; 7.680(2 \mathrm{H}, d, J=16 \mathrm{~Hz}, \mathrm{C} 7,11$, aliphatic-CH); dan $7.883(2 \mathrm{H} d, J=2 \mathrm{~Hz}, \mathrm{C} 6,17, \mathrm{Ar}-$ CH. ${ }^{13} \mathrm{C}-\mathrm{NMR}\left(125 \mathrm{MHz}, \mathrm{ppm}, \mathrm{CDCl}_{3}\right): \delta 111.696, \delta 117.609, \delta 124.789, \delta 129.473, \delta 130.634, \delta 134.781$, $\delta 143.890, \delta 158.116, \delta 191.365$.

\section{1,5-bis-(2'-kloro-6'-fluoro benzylidene)-1,4-pentadien-3-one (C02)}

Light yellow powder, yield $63 \%, \mathrm{mp} 142.2-143.2^{\circ} \mathrm{C} ; \mathrm{Rf}=0.35$, ethyl acetate : $\mathrm{n}$-hex(1:9); IR $\gamma(\mathrm{cm}-1)$ $(\mathrm{KBr}): 3126.28$ (=C-H stretching aromatic), 3050,55 ( $\mathrm{C}-\mathrm{H}$ stretching aliphatic), $1699.46(\mathrm{C}=\mathrm{C}$ stretching aliphatic), $1633.22\left(\mathrm{C}=\mathrm{O}\right.$ stretching $\alpha \beta, \alpha{ }^{\prime} \beta^{\prime}$-un-sat $) ; 1453.41(\mathrm{C}=\mathrm{C}$ stretching aromatic $), 1368.24(\mathrm{C}-\mathrm{H}$ bending aliphatic), 1228.33 (C-CO-C coupled stretching and bending), 1018.46 (C-F stretching), 687.71

(C-Cl stretching); MS (EI-MS, m/z ): $339\left[\mathrm{M}^{+}+2 \mathrm{H}\right] ; 118$ (base peak) $\left[\mathrm{M}^{+\cdot}\right]$; 339, 303, 277, 209, 183, $155,130,89,63 ;{ }^{1} \mathrm{HNMR}\left(500 \mathrm{MHz}, \mathrm{ppm}, \mathrm{CDCl}_{3}-\mathrm{d} 6\right): \delta 7.027(2 \mathrm{H}, d d, J 1=J 2=5 \mathrm{~Hz}, \mathrm{C} 2,15,-\mathrm{CH}$ aliphatic), $\delta 7.231(4 \mathrm{H}, m, \mathrm{C} 1,3,14,16, \mathrm{Ar}-\mathrm{CH}), \delta 7.300(2 \mathrm{H}, d, J=17 \mathrm{~Hz}, \mathrm{C} 8,10, \mathrm{Ar}-\mathrm{CH})$, dan $\delta 7.911(2 \mathrm{H}$, $d, J=16.5 \mathrm{~Hz} \mathrm{C} 7,11, \mathrm{CH}$ aliphatic); ${ }^{13} \mathrm{C}-\mathrm{NMR}\left(125 \mathrm{MHz}, \mathrm{ppm}, \mathrm{CDCl}_{3}\right): \delta 115.212, \delta 122.085, \delta 122.200$, $\delta 126.279, \delta 131.923, \delta 133.920, \delta 161.353, \delta 163.282, \delta 189.506$.

\section{In-vitro Antibacterial Assay Microdilution Method}

$50 \mu \mathrm{L}$ of assay solutions and $50 \mu \mathrm{L}$ of bacterial included in 1 well, similar treatment was also carried out on control. Media control contains $100 \mu \mathrm{L}$ of media only, whereas the control compound contains $50 \mu \mathrm{L}$ of test compound and $50 \mu \mathrm{L}$ of media. The microplate then incubated for 24 hours at $37^{\circ} \mathrm{C}-$ the minimum inhibitory value (MIC) determined by observing the clarity of the solution. The minimum bactericidal concentration (MBC) determination is done by scratching the MIC results on the Mueller Hinton agar (MHA) medium, then incubated for 24 hours at $37^{\circ} \mathrm{C}$. Besides, the absorbance of the solution on each well on the microplate was carried out using a microplate reader at $595 \mathrm{~nm}$ wavelength to determine the percent inhibition of bacterial growth from the total population. The percent inhibition results obtained were then calculated $\mathrm{IC}_{50}$ value of each compound.

Agar Diffusion Method

Fifteen $\mathrm{mL}$ of Mueller Hinton agar (MHA) added to the petri dish, then added $50 \mu \mathrm{L}$ of bacterial suspension (McFarland standard 3, $9.0 \times 108)$. Make a well on the media with the available tools $(\Theta$ $6 \mathrm{~mm})$. Put $20 \mu \mathrm{L}$ of the test compound in the well, then incubate at $37^{\circ} \mathrm{C}$ for 24 hours. Measure the clear/ inhibitory zone on the well.

\section{RESULTS AND DISCUSSION}

The analogs mono-carbonyl of curcumin have been successfully synthesized and pure. Purity test was carried out using HPLC. The antibacterial assay carried out based on CLSI (Clinical and Laboratory Standards Institute) 2012 guidelines with some modifications against six bacteria and one yeast. Positive controls used in this study were amoxicillin, chloramphenicol, kanamycin, and streptomycin. The basis for the selection of positive controls is a consideration of pharmacological effects. Microdilution is an antibacterial assay method with a principle similar to the liquid dilution method but uses a 96 wells microplate device with small amounts of compounds, media, and bacteria (total volume of $100 \mu \mathrm{L}$ ). The advantage of this method is that it is more sensitive, effective, and more efficient because it can test many 
RASĀYAN $J$. Chem.

Vol. 13 | No. 2 |1153 - 1165| April - June | 2020

samples in a short amount of time. ${ }^{15}$ MIC describes the ability of compounds to inhibit bacterial growth, $\mathrm{MBC}$ describes the ability of compounds to kill bacteria, and $\mathrm{IC}_{50}$ values describe the ability of compounds to inhibit bacterial growth by 50 percent for 24 hours.

Microdilution assay results show that the compound of A01, B01, C01 and $\mathrm{C} 02$ has the potential to inhibit the growth of bacteria mainly in gram-positive and several gram-negative bacteria. Test results show that there is a correlation between the concentrations of the test compound with the percentage of inhibition of all test bacteria. The higher concentration gives more significant inhibitory activity than a smaller concentration. The ability of compounds to inhibit microbial growth shown in Fig.-2.
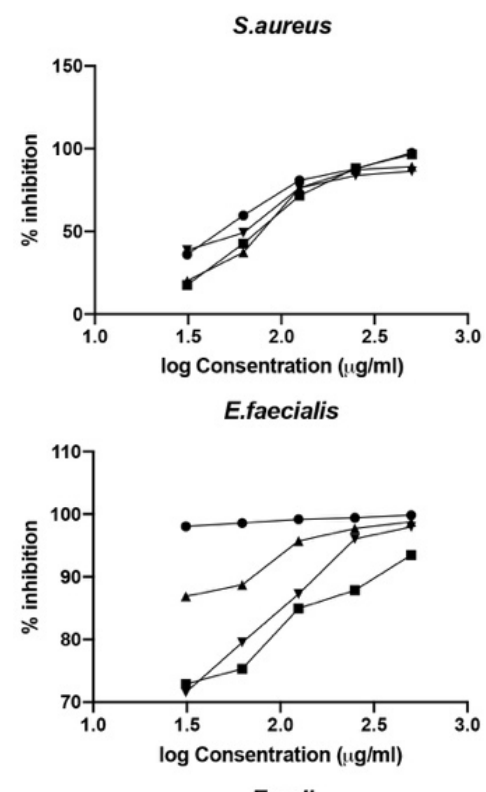

E.coli

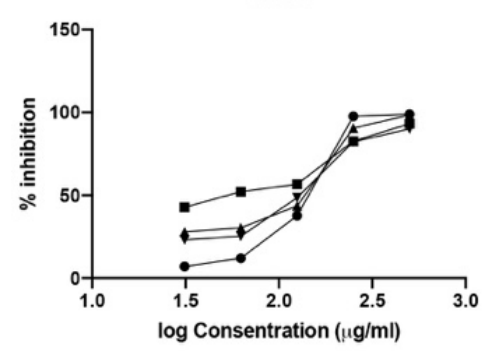

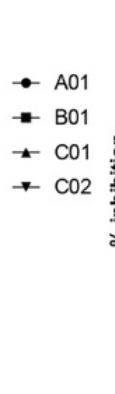
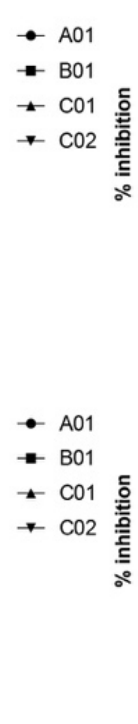

B.subtilis

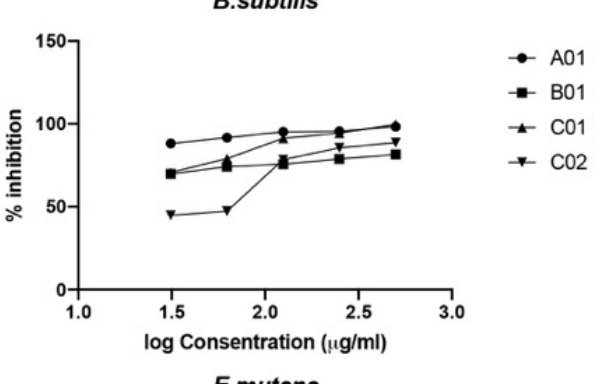

E.mutans

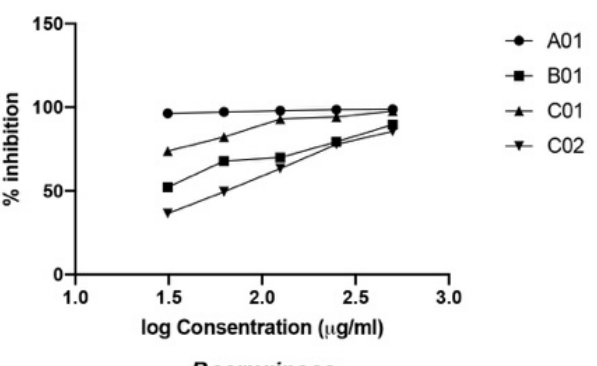

P.aeruginosa

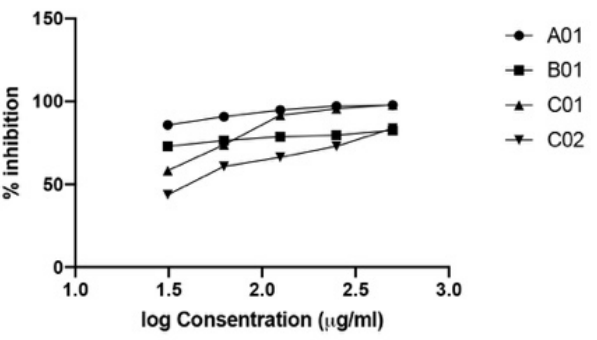

C.albicans

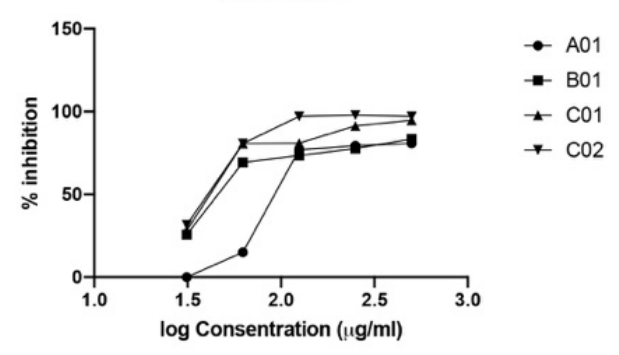

Fig.-2. Percent Inhibition of Test Compounds against Bacteria

In $31.25 \mu \mathrm{g} / \mathrm{mL}$, compound $\mathrm{A} 01, \mathrm{~B} 01$, and $\mathrm{C} 01$ gave a considerable percent inhibitory value against $\mathrm{B}$. subtilis, S. mutans, E. faecialis, and P. aeruginosa. A01 gave the lowest percent inhibition against E.coli, while $\mathrm{B} 01$ and $\mathrm{C} 01$ gave the lowest inhibition against $\mathrm{S}$. aureus. The $\mathrm{C} 02$ compound gives a substantial percent inhibition value against E.faecialis at a concentration of $31.25 \mu \mathrm{g} / \mathrm{mL}$, while the lowest percent 
RASĀYAN J. Chem.

Vol. 13 | No. 2 |1153 - 1165| April - June | 2020

inhibition against E. coli. So it can be concluded that the compounds A01, B01, and C01 can inhibit the same bacteria, namely B. subtilis, S. mutans, E.faecialis, and P. aeruginosa. All test compounds gave low inhibitory activity against $\mathrm{E}$. coli bacteria. Test compounds showed different results in antifungal activity assay. Antifungal assay results do not provide a good correlation between concentration and inhibitory ability, although it has the good inhibitory ability at high concentrations. MIC values of the test compounds shown in Table-2.

Table-2: The MIC Value of the Test Compound against Test Bacteria

\begin{tabular}{|c|c|c|c|c|c|c|c|}
\hline \multirow{3}{*}{ Comp. } & \multicolumn{7}{|c|}{$\mathrm{MIC}(\mu \mathrm{g} / \mathrm{mL})$} \\
\hline & \multicolumn{4}{|c|}{ Gram-positive Bacteria } & \multicolumn{2}{|c|}{ Gram-negative Bacteria } & \multirow{2}{*}{$\begin{array}{c}\text { Yeast } \\
\text { C.albicans }\end{array}$} \\
\hline & S.aureus & B.subtilis & E.faecialis & S.mutans & E.coli & P.aeruginosa & \\
\hline A01 & 50,3 & 50,3 & 50,3 & 100,5 & 402,0 & 100,5 & 200,98 \\
\hline B01 & 1710,4 & 1710,4 & 1710,4 & $-*$ & 1710,4 & $-*$ & 427,6 \\
\hline $\mathrm{C} 01$ & 294,8 & 294,8 & 589,5 & 294,8 & 1179 & 294,8 & 294,75 \\
\hline $\mathrm{C} 02$ & 1474,2 & 1474,2 & 1474,2 & $-*$ & 1474,2 & $-*$ & 368,55 \\
\hline Am. & 42,8 & 42,8 & 42,8 & 42,8 & 42,8 & 42,8 & - \\
\hline K1. & 24,1 & 24,1 & 24,1 & 24,1 & 24,1 & 24,1 & - \\
\hline $\mathrm{Ka}$. & 16,1 & 16,1 & 258,0 & 16,1 & 258,0 & 16.1 & - \\
\hline Strep. & 13,4 & 13,4 & 214,9 & $-*$ & 429,87 & 13,4 & - \\
\hline
\end{tabular}

$\mathrm{Nb}$ : sign (-*) indicates that the compound has no inhibitory activity (MIC). (-) indicates no test performed. Am. (Amoxicillin), Kl. (Chloramfenicol), Ka. (Kanamycin), Strep. (Streptomycin)

The best MIC value of the test compound is a test compound with the ability to inhibit bacterial growth for 24 hours at low concentrations. A01 is the most potent compound against $S$. aureus, B. subtilis, E.faecialis, S. mutant, and P.aeruginosa, as shown in Table-2. Based on the positive MIC, were scratch on the MHA media to obtain MBC values, as shown in Table-3.

Table-3: The MBC Value of the Test Compound against Test Bacteria

\begin{tabular}{|c|c|c|c|c|c|c|c|}
\hline \multirow{3}{*}{ Comp. } & \multicolumn{7}{|c|}{$\operatorname{MBC}(\mu \mathrm{g} / \mathrm{mL})$} \\
\hline & \multicolumn{4}{|c|}{ Gram-positive Bacteria } & \multicolumn{2}{|c|}{$\begin{array}{c}\text { Gram-negative } \\
\text { Bacteria }\end{array}$} & \multirow{2}{*}{$\begin{array}{c}\text { Yeast } \\
\text { C.albicans }\end{array}$} \\
\hline & S.aureus & B.subtilis & E.faecialis & S.mutans & E.coli & P.aeruginosa & \\
\hline A01 & 50,3 & 50,3 & 50,3 & 100,49 & $-*$ & 100,5 & $-*$ \\
\hline B01 & $-*$ & $-*$ & $-*$ & $-*$ & $-*$ & $-*$ & $-*$ \\
\hline $\mathrm{C01}$ & 294,8 & 294,8 & 589,5 & 294,8 & 1179,0 & $-*$ & $-*$ \\
\hline $\mathrm{C} 02$ & $-*$ & $-*$ & $-*$ & $-*$ & $-*$ & $-*$ & $-*$ \\
\hline Am. & 42,8 & 42,8 & 42,8 & 42,8 & 42,8 & 42,8 & \\
\hline $\mathrm{K} 1$. & 24,1 & 24,1 & 24,1 & 24,1 & 24,1 & 24,1 & - \\
\hline Ka. & 16,1 & 16,1 & 258,0 & 16,1 & 258,0 & 16,1 & - \\
\hline Strep. & 13,4 & 13,4 & 214,9 & $-*$ & 214.9 & 13,4 & - \\
\hline
\end{tabular}

$\mathrm{Nb}$ : sign $\left(-^{*}\right)$ indicates that the compound has no inhibitory activity (MIC). (-) indicates no test performed. Am. (Amoxicillin), Kl. (Chloramfenicol), Ka.(Kanamycin), Strep.(Streptomycin)

Not all compounds that have positive MIC values give MBC values. In A113, MBC values were positive for almost all test bacteria except for S. mutants and E. coli. The media shows the growth of bacterial colonies. In $\mathrm{C} 114$, also gave a positive $\mathrm{MBC}$ value to almost all test bacteria except for $\mathrm{B}$. subtilis and $\mathrm{P}$. aeruginosa. At the same time, B146 and C143 showed that none of the positive MIC values gave a positive $\mathrm{MBC}$ value as well. $\mathrm{IC}_{50}$ values of each test compound can be determined after knowing the percent inhibition value, MIC value, and KBM, as shown in Table-4.

Based on statistical analysis, antibacterial activity of all test compounds (A01, B01, C01, and C02) against all test bacteria showed no significant difference $(p=0.083 ; 0.083 ; 0.446 ; 0.083 ; 0.261 ; 0.104)$. In addition to the microdilution method, the antibacterial assay also carried out with the agar diffusion method. This method is similar to the disk diffusion method (Kirby-Bauer method). The clear zone of an 
RASĀYAN J. Chem.

Vol. 13 | No. 2 |1153 - 1165| April - June | 2020

area at the well shows its inhibitory activity. Inhibition zone diameter of the test compound against test bacteria shown in Table-5.

Table-4. The $\mathrm{IC}_{50}$ value of the test compound against test bacteria

\begin{tabular}{|c|c|c|c|c|c|c|c|}
\hline \multirow{3}{*}{ Comp } & \multicolumn{7}{|c|}{$\mathrm{IC}_{50}(\mu \mathrm{M})$} \\
\hline & \multicolumn{4}{|c|}{ Gram-positive Bacteria } & \multicolumn{2}{|c|}{ Gram-negative Bacteria } & \multirow{2}{*}{$\begin{array}{c}\text { Yeast } \\
\text { C.albicans }\end{array}$} \\
\hline & S.aureus & B.subtilis & E.faecialis & S.mutant & E.coli & P.aeruginosa & \\
\hline $\mathrm{A} 01$ & $37,1 \pm 4,89$ & $56,8 \pm 5,47$ & $\begin{array}{c}97,2 \pm \\
82,81\end{array}$ & $66.6 \pm 30,53$ & $291,2 \pm 0,91$ & $29,6 \pm 6,79$ & $44,8 \pm 0,00$ \\
\hline B01 & $139 \pm 3,07$ & $\begin{array}{c}288,9 \pm \\
21,72\end{array}$ & $\begin{array}{c}392,2 \pm \\
13,79\end{array}$ & $\begin{array}{c}328,6 \pm \\
24,38\end{array}$ & $1026 \pm 0,73$ & $171,2 \pm 15,63$ & $107-213,8$ \\
\hline $\mathrm{C} 01$ & $79,0 \pm 0,54$ & $98.8 \pm 6,43$ & $\begin{array}{c}129,7 \pm \\
0.25\end{array}$ & $49,2 \pm 1,35$ & $679,8 \pm 4.00$ & $33,0 \pm 0,28$ & $73,7-147,4$ \\
\hline $\mathrm{C} 02$ & $\begin{array}{c}117,1 \pm \\
3,32\end{array}$ & $\begin{array}{c}186,1 \pm \\
8,71\end{array}$ & $\begin{array}{c}191,6 \pm \\
79.08\end{array}$ & $\begin{array}{c}253,8 \pm \\
28,14\end{array}$ & $562 \pm 0,21$ & $130,6 \pm 26,86$ & $92,1-184,3$ \\
\hline Am. & $26,5 \pm 0,00$ & $17,2 \pm 4,42$ & $16,7 \pm 3,96$ & $14,0 \pm 12,73$ & $71,2 \pm 13,86$ & $15,1 \pm 11,1$ & - \\
\hline K1. & $20,3 \pm 0,00$ & $26,3 \pm 5,02$ & $9,6 \pm 1,98$ & $7,9 \pm 5,51$ & $3,8 \pm 0,99$ & $15,9 \pm 1,84$ & - \\
\hline Ka. & $17,4 \pm 1,32$ & $19,5 \pm 2,83$ & $92,2 \pm 5,66$ & $24,5 \pm 5,65$ & $11,1 \pm 4,24$ & $17,7 \pm 12,01$ & - \\
\hline Strep. & $14.7 \pm 0,00$ & $19.9 \pm 0,00$ & $155 \pm 0,00$ & $23.3 \pm 0,00$ & $22.4 \pm 0,00$ & $23.8 \pm 0,00$ & - \\
\hline
\end{tabular}

$\mathrm{Nb}$ : sign (-) indicates no test performed. Am. (Amoxicillin), Kl. (Chloramfenicol), Ka.(Kanamycin), Strep.(Streptomycin)

Table-5: The Inhibition Zone Diameter of the Test Compound against Test Bacteria

\begin{tabular}{|c|c|c|c|c|c|c|c|}
\hline \multirow{3}{*}{ Comp. } & \multicolumn{7}{|c|}{ Inhibition Zone Diameter (mm) } \\
\hline & \multicolumn{4}{|c|}{ Gram-positive Bacteria } & \multicolumn{2}{|c|}{$\begin{array}{c}\text { Gram-negative } \\
\text { Bacteria }\end{array}$} & \multirow{2}{*}{$\begin{array}{c}\text { Yeast } \\
\text { C.albicans }\end{array}$} \\
\hline & S.aureus & B.subtilis & E.faecialis & S.mutant & E.coli & P.aeruginosa & \\
\hline A01 & 9 & 13 & 12 & 17 & $-*$ & 18 & $-*$ \\
\hline B01 & $-*$ & $-*$ & $-*$ & $-*$ & $-*$ & $-*$ & $-*$ \\
\hline $\mathrm{C} 01$ & 8 & 8 & 16 & 10 & $-*$ & 7 & $-*$ \\
\hline $\mathrm{C} 02$ & 8 & $-*$ & $-*$ & $-*$ & $-*$ & $-*$ & $-*$ \\
\hline Am. & 31 & 10 & - & 21 & 17 & 18 & \\
\hline Kl. & 25 & 27 & 20 & 32 & 35 & 28 & - \\
\hline Ka. & 26 & 21 & 5 & 24 & 18 & 22 & - \\
\hline Strep. & 22 & 20 & - & 16 & 16 & 21 & - \\
\hline
\end{tabular}

Nb: sign (-) indicates no test performed. Am. (Amoxicillin), Kl. (Chloramfenicol), Ka.(Kanamycin), Strep.(Streptomycin). The concentration of compound test $1000 \mu \mathrm{g} / \mathrm{mL}$

Drug control concentration $500 \mu \mathrm{g} / \mathrm{mL}$

Well diameter $4 \mathrm{~mm}$

The results showed that only compounds $\mathrm{A} 01$ and $\mathrm{C} 01$ gave proper inhibition diameters for Grampositive bacterial strains, whereas Gram-negative bacterial strains did not show clear zones, as shown in table-5. This result ties well with previous studies wherein that the curcumin analog is more sensitive to gram-positive bacteria. ${ }^{1,3,4,9}$

\section{Study Docking}

In docking studies were carried out to confirm and to find out the mechanism of action of the synthesized compounds as an antibacterial, which had previously proven in vitro. In study docking used 1PCW as a protein test, which is a protein from D-Alanyl-D-Alanine Carboxypeptidase and 3N7W, which is a protein from beta-lactamase. The selection of proteins above based on the previous study, which reported that mono-carbonyl analogs have antibacterial activity through inhibition of bacterial cell wall biosynthesis.

Before the docking process begins, we need to prepare the previously downloaded protein in the data bank. The first step in a docking study is to choose one of the chains in the target protein and clean the 
RASĀYAN J. Chem.

Vol. 13 | No. 2 |1153 - 1165| April - June | 2020

protein from residue. This process uses MOE 2018.01.01 software. Discarded residues such as water molecules and metal residues. After the protein is free from the residue, the next preparation is to add hydrogen atoms, add atomic charges, and repair the bonds of atoms damaged by the release of water molecules and metal residues. After the preparation process is complete, the protein is ready to validate.

The protocol of validation protein performed using the Alpha PMI method on placement, Induced fit on refinement, while the scoring was using the GBVI/ WSA dG method. The validity of the protein assessed from the value of RMSD (Root Mean Square Deviation). RMSD is a parameter used to evaluate the similarity of two structures. The similarity is measured based on differences in distances of similar atoms. Validation results show that the value of RMSD on 1PWC and 3N7W proteins are 1.5838 and 1.9087, respectively. Validation result, as shown in Fig.-3.
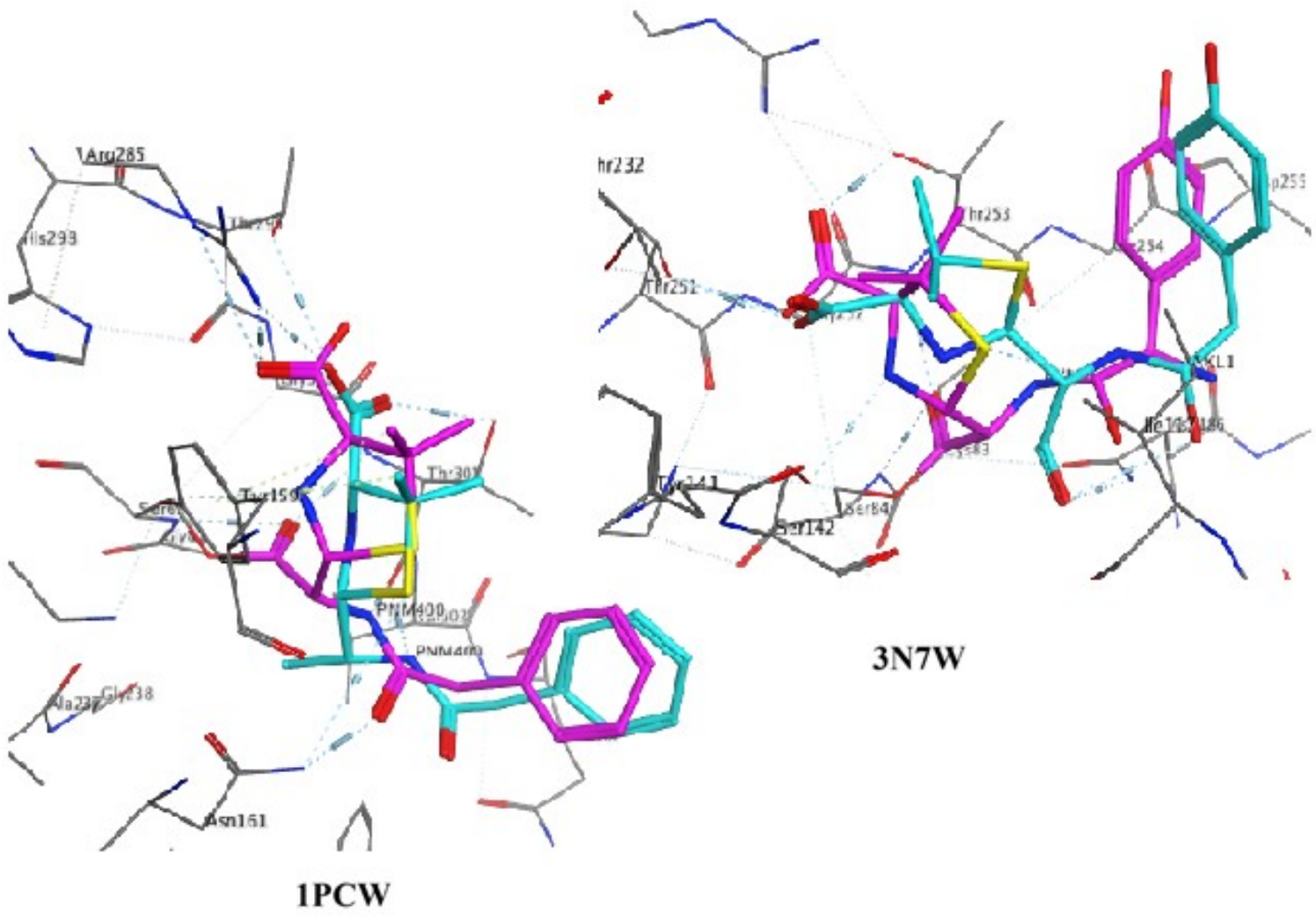

Fig.-3: Confirmation of the Results of the Validation

The result docking studies on the 1PWC protein (as shown in Fig.-4. ) it is known that the key receptors / amino acid residues that play an essential role in D-Alanyl-D-Alanine Carboxypeptidase are THR301, SER62, THR299, ARG285, and ASN16 which known from the interaction between native ligands and proteins both 2D and 3D. In $\mathrm{A} 01$, the $\mathrm{Br}(4)$ interacts with the $\mathrm{O}$ atom from THR 123 through the Hdonor, the $\mathrm{Br}$ atom (38) interacts with the $\mathrm{O}$ atom from THR299 through the $\mathrm{H}$-donor, and the $\mathrm{O}$ atom (41 from the ketone) interacts with the $\mathrm{N}$ atom from ASN161 through $\mathrm{H}$-acceptor. In B01, the $\mathrm{O}$ atom (34, from the hydroxyl group) interacts with the $\mathrm{C}$ atom of SER62 via the $\mathrm{H}$-donor, while the $\mathrm{O}$ atom (38, of the ketone) interacts with the $\mathrm{N}$ atom of ASN161 through the H-acceptor. The aromatic group of B01 (6ring) interacts with the aromatic group (6-ring) of TYR159 through pi-pi bonds. Atom O (34, ketone group) of $\mathrm{C} 01$ interacts with atom $\mathrm{N}$ of ASN161 through $\mathrm{H}$-acceptor. In $\mathrm{C} 02$, the $\mathrm{O}$ atom (32, from the ketone) interacts with the $\mathrm{N}$ atom from ASN161 through $\mathrm{H}$-acceptor, while the aromatic group (6-ring) C02 interacts with the aromatic (6-ring) group of TYR159 through pi-pi.

The results of in silico studies on the $3 \mathrm{~N} 7 \mathrm{~W}$ protein found that key receptors / amino acid residues that play an essential role in the activity of the beta-lactamase enzyme are SER84, THR253, THR251, LYS250, and ARG236 (as shown in Fig.-5). In A01, the C atom (1, from the aromatic ring) has an 
RASĀYAN $J$. Chem.

Vol. 13 | No. 2 |1153 - 1165| April - June | 2020

interaction with the $\mathrm{C}$ atom of SER84 through $\mathrm{H}$-donor. The $\mathrm{O}$ atom of ILE139 has interactions with The $\mathrm{Br}$ atom (4, from the aromatic ring) the $\mathrm{O}$ atom of ILE139 through $\mathrm{H}$-donor. The $\mathrm{Br}$ atom (4, from the aromatic ring) has interactions with the $\mathrm{O}$ atom of ASN230 through the $\mathrm{H}$-donor. $\mathrm{O}$ atom (6, from the hydroxyl group) has an interaction with $\mathrm{O}$ atom of THR251 through H-donor. In B01, the O atom (34, from the hydroxyl group in the aromatic ring) has an interaction with the $\mathrm{C}$ atom of SER84 through $\mathrm{H}$ donor. In $\mathrm{C} 01$, the $\mathrm{O}$ atom (6, from the hydroxyl group on the aromatic ring) has an interaction with the $\mathrm{O}$ atom of ASP255 through $\mathrm{H}$-donor. In contrast, the $\mathrm{O}$ atom (28, from the hydroxyl group on the aromatic ring) has an interaction with the $\mathrm{O}$ atom from SER84 through $\mathrm{H}$ - donor and THR251 through $\mathrm{H}$-acceptor. In the $\mathrm{C} 02, \mathrm{Cl}$ atom (10, from the aromatic group) has an interaction with the $\mathrm{C}$ atom on SER84 through $\mathrm{H}$-donor.
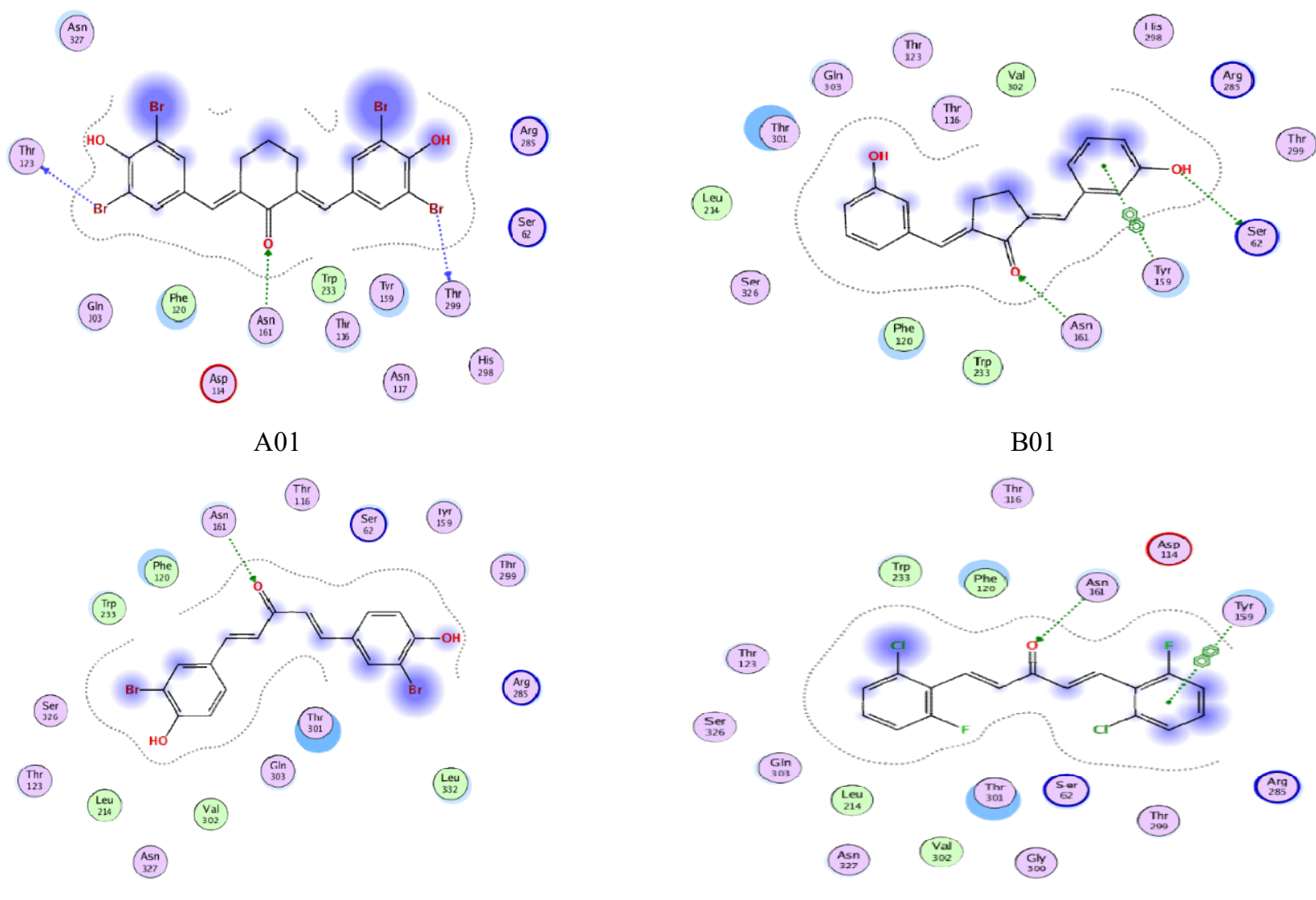

(Tir)
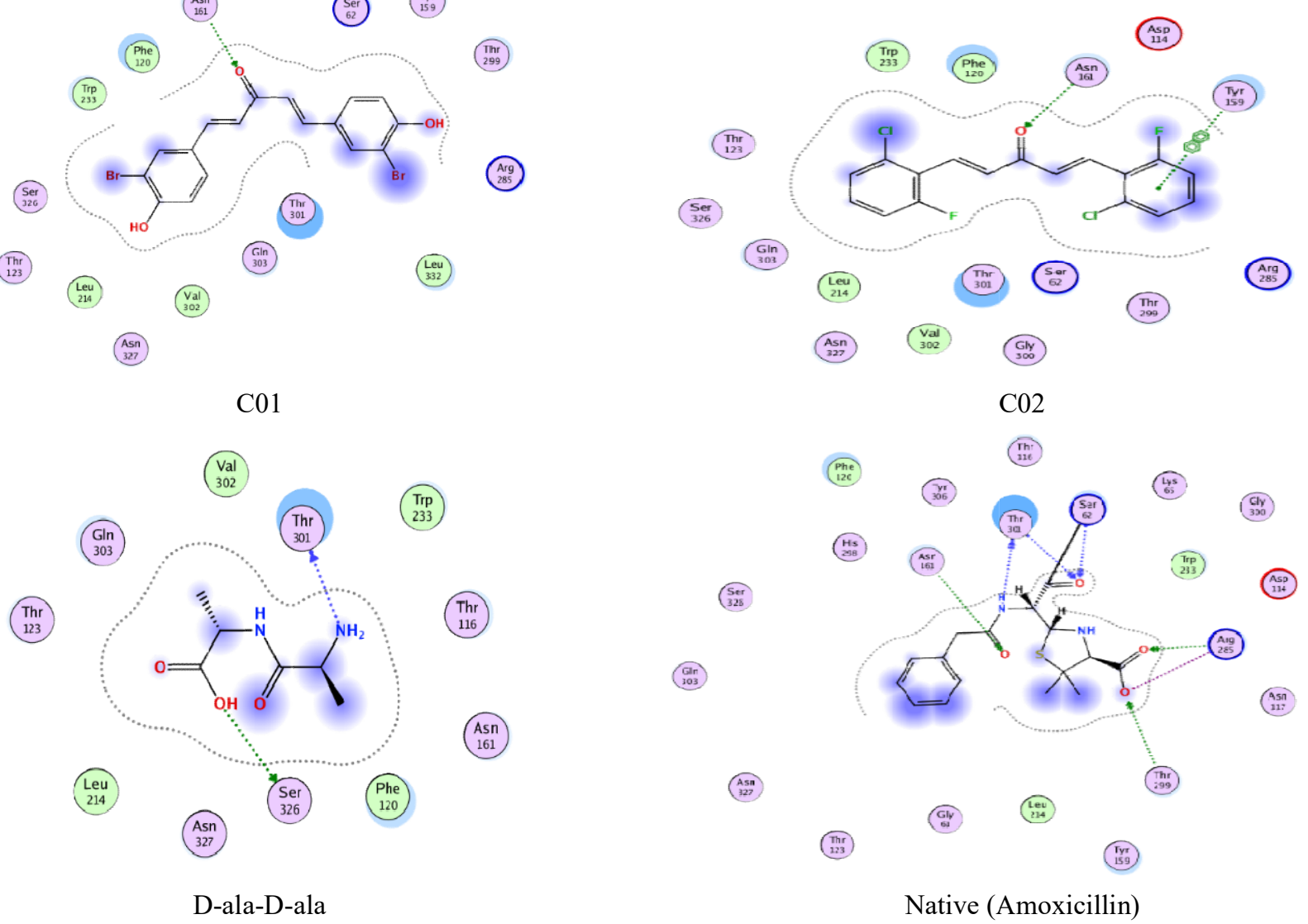

D-ala-D-ala

Fig.-4: Interaction of Test Compound on 1PWC

1162 
RASĀYAN J. Chem.

Vol. 13 | No. 2 |1153 - 1165| April - June | 2020

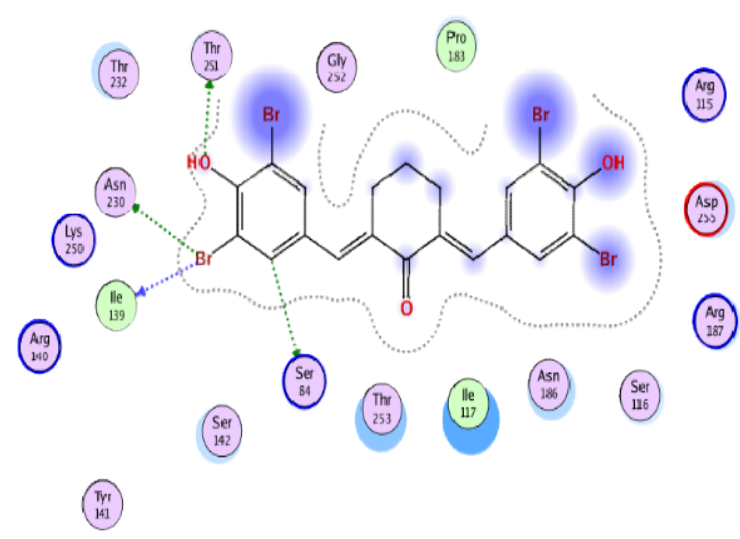

A01

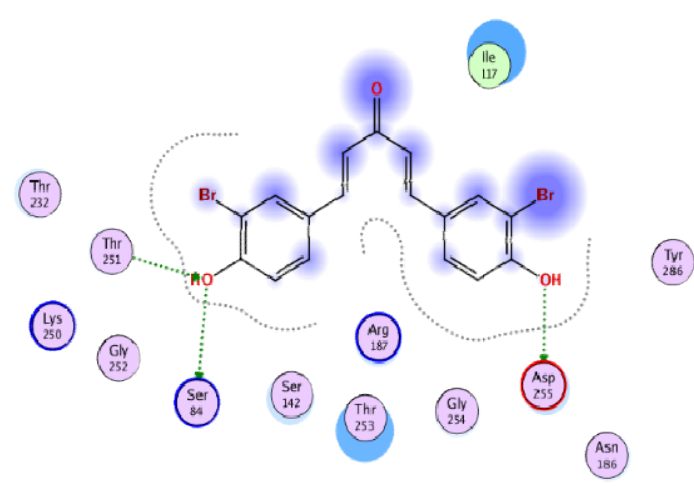

(A99)

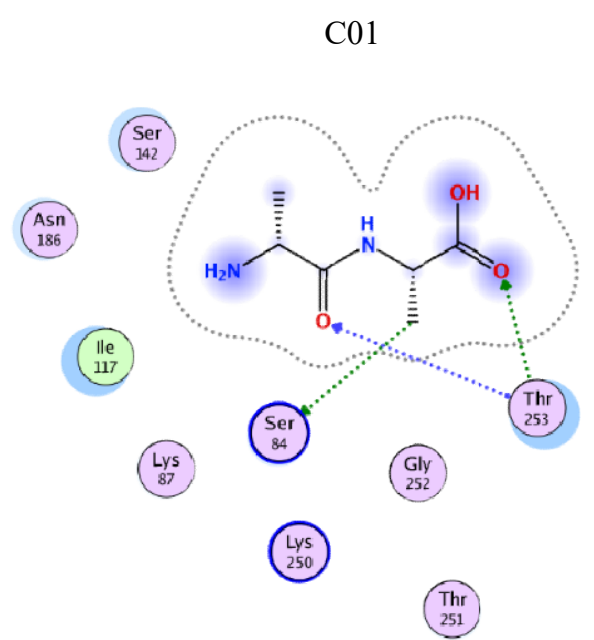

D-ala-D-ala

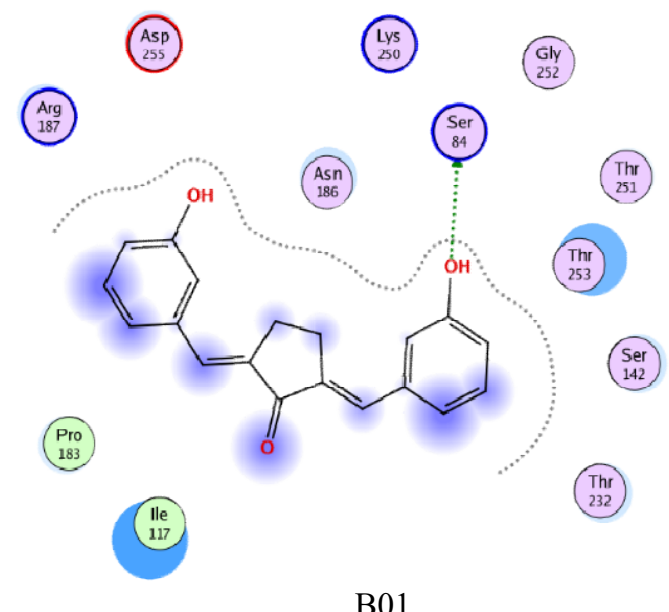

(GIY)

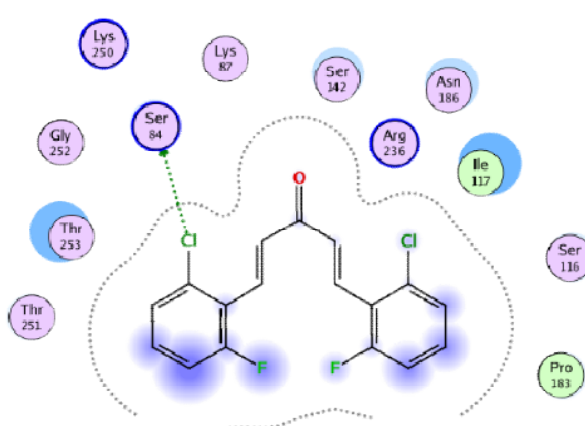

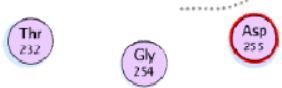

$\mathrm{C} 02$

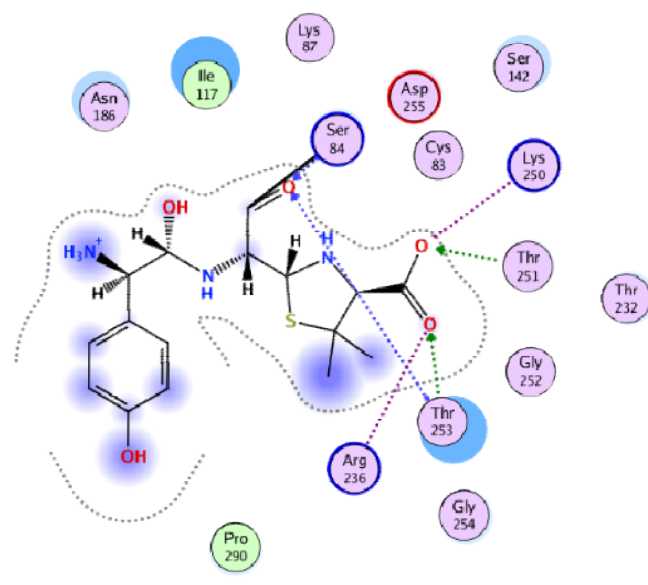

Native (Amoxicillin)

Fig.-5: Interaction of Test Compound on 3N7W

After docking prose and known interactions that occur between the test compound (Test ligand) with the target proteins, the $\mathrm{S}$ value of each test ligand, also known as shown in table-6. S value is the value that describes the strength of the bond affinity that occurs between the test ligand and the target protein. The 
RASĀYAN J. Chem.

Vol. 13 | No. 2 |1153 - 1165| April - June | 2020

smaller the value of $\mathrm{S}$, the higher the affinity and stable bonds that occur, so the more significant the activity.

Table-6: Docking Score of Ligand Test

\begin{tabular}{c|c|c}
\hline \multirow{2}{*}{ Comp. } & \multicolumn{2}{|c}{ Docking Score (S) } \\
\cline { 2 - 3 } & 1PWC & 3N7W \\
\hline A01 & -5.6910 & -5.5275 \\
\hline B01 & -5.7984 & -5.4582 \\
\hline C01 & -6.0139 & -5.4546 \\
\hline C02 & -5.6095 & -5.6920 \\
\hline D-Ala-D-ala & -4.4585 & -4.2879 \\
\hline Native (amoxicillin) & -6.4361 & -6.5012 \\
\hline
\end{tabular}

The result is consistent with what has found in previous that mono-carbonyl curcumin compounds have antibacterial activity through the mechanism of cell wall biosynthesis ${ }^{9}$ inhibition that has proven through docking studies. The two main structures responsible for its antibacterial activity are the substituents of symmetrical aromatic benzene and $\alpha, \beta$-unsaturated ketones (Enone groups) that bind to the active site of the receptor site of action as shown in Fig.-6.

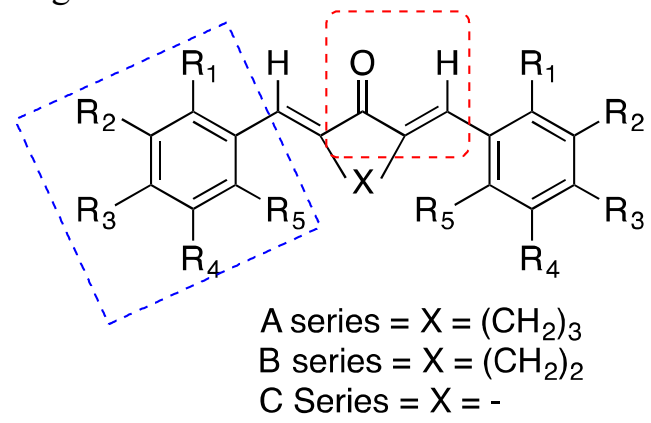

-- - substituent of benzene aromatic

-..- $\alpha, \beta$-unsaturated (Enone groups)

Fig.-6: The Binding Site of Mono-carbonyl Analog of Curcumin

\section{CONCLUSION}

Study results show that the mono-carbonyl curcumin analog compound has antibacterial activity through inhibition of bacterial cell wall synthesis.

\section{ACKNOWLEDGMENT}

The authors are grateful to the Medicinal Chemistry Department, Faculty of Pharmacy, and Universitas Gadjah Mada, Yogyakarta, Indonesia for the license of MOE 2018.01.01 and Universitas Tanjungpura for supported this study.

\section{REFERENCES}

1. A. Singh, J.V. Singh, A. Rana, K. Bhagat, H.K. Gulati, R. Kumar, R. Salwan, K. Bhagat, G. Kaur, N. Singh, R. Kumar, H. Singh, S. Sharma, and P.M.S. Bedi, American Chemical Society Omega, 4, 11673(2019), DOI:10.1021/acsomega.9b01109

2. C. Dye, Philosophical Transactions of The Royal Society B Biological Sciences, 369, 20130426(2014), DOI:10.1098/rstb.2013.0426

3. M. Idress, S. Kola, and N. J. Siddiqui, Rasayan Journal of Chemistry, 12(4), 1725(2019), DOI:10.31788/RJC.2019.1245467

4. M. Shivanand, P.Vijaya Kumar, and V. Ravikumar, Rasayan Journal of Chemistry, 12(4), 1810(2019), DOI:10.31788/RJC.2019.1245375 
RASĀYAN J. Chem.

Vol. 13 | No. 2 |1153 - 1165| April - June | 2020

5. P. Kumar, S.K. Kandi, S. Manohar, K. Mukhopadhyay, and D.S. Rawat, American Chemical Society Omega 4, 675(2019), DOI:10.1021/acsomega.8b02625

6. G. Liang, L. Jiang, S. Yang and Y. Zhao. Chemical \& Pharmaceutical Bulletin, 56, 162(2008), DOI: 10.1248/cpb.56.162

7. P.R. Baldwin, A.Z. Reeves, K.R. Powell, R.J. Napier, A.I. Swimm, A. Sun, K. Giesler, B. Bommarius, T.M. Shinnick, J.P. Synder, D.C. Liotta, and D. Kalman, European Journal of Medicinal Chemistry, 92, 693(2015), DOI:10.1016/j.ejmech.2015.01.020

8. C. Carapina da Silva, B.S. Pacheco, R.N. das Neves, M.S. Dié Alves, A. Sena-Lopes, S. Moura, S. Borsuk, and C.M.P. de Pereira, Biomedicine Pharmacotheraphy, 111, 367(2019), DOI: 10.1016/j.biopha.2018.12.058

9. M. Reksohadiprodjo, et al. US patent US203/09272A1 (2003),

10. I.M. Fawzy, K.M. Youssef, N.S.M. Ismail, J. Gullbo, and K.A.M Abouzid, Future Journal of Pharmaceutical Sciences, 1, 22(2015), DOI:10.1016/j.fjps.2015.06.001

11. Sardjiman. Synthesis of Some New Series of Curcumin Analogs, Antioxidative, Antiinflamatory, Antibacterial Activities, and Qualitative Structure-Activity Relationships, Universitas Gadjah Mada, 2000 .

12. S.S. Sardjiman, M.S. Reksohadiprodjo, L. Hakim, H. Van der Goot and H. Timmerman, European Journal of Medicinal Chemistry, 32, 625(1997), DOI: 10.1016/S0223-5234(97)83288-6

13. A. Vyas, P. Dandawate, S. Padhye, A. Ahmad and F. Sarkar, Current Pharmaceutical Design, 19, 2047(2013), DOI: 10.2174/1381612811319110007

14. C. Selvam, S.M Jachak, R. Thilagavathi and A.K. Chakraborti, Bioorganic and Medical Chemistry Letter, 15, 1793(2005), DOI:10.1016/j.bmcl.2005.02.039

15. F. Dong, C. Jian, F. Zhenghao, G. Kai, and L. Zuliang, Catalysis Communications, 9, 1924(2008), DOI: $10.1016 /$ j.catcom.2008.03.023

16. L. Claisen, and A. Claparède, Berichte der deutschen chemischen Gesellschaft, 14, 2460(1881), DOI: $10.1002 /$ cber. 188101402192

17. G. Brooks, K.C. Carroll, J. Butel and S. Morse, Jawetz Melnick \& Adelbergs Medical Microbiology 26/E, McGraw-Hill Publishing, 2012

[RJC-5554/2019] 